\title{
Modelling technique of interaction between pressure and gravity sewage systems
}

\author{
Ireneusz Nowogoński ${ }^{1, *}$, and Ewa Ogiołda ${ }^{1}$ \\ ${ }^{1}$ University of Zielona Góra, Faculty of Civil Engineering, Architectural and Environmental \\ Engineering, 65-417 Zielona Góra, Poland
}

\begin{abstract}
The article presents the issue of cooperation between gravity and pressure elements realized in a common simulation model. The analysis was based on examples characterized by different levels of complexity. It was found that the SWMM model is possible to apply for the analysis of complete gravity-pressure systems, even in the event of a few pumping stations pumping to a common pressure pipeline. The possibility of applying models prepared in such a manner for the assessment of the correctness of pump selection in terms of the time sewage is stored and frequency of the pump starting up.
\end{abstract}

\section{Introduction}

The modelling of gravity-pressure sewage and rainwater discharge systems requires the application of diametrically different calculation algorithms. The choice of programme enabling the analysis of pressure components depends on the level of complexity characterizing the system. Among others, the length of the pressure pipeline as well as the relief of the terrain are important. The choice is also influenced by the occurrence of connections of pressure pipelines into complicated systems, which may be characterized by the simultaneous operation of a few stations pumping sewage to a common pressure pipeline.

\section{Method}

It is easiest to model entirely pressure-based or gravity-based water sewage systems. Introducing elements of a mixed system complicates the situation and requires an individual approach, depending on the level of complexity characterizing the given system [1]. Typical examples have been presented graphically in Figures 1 - single pumping station allowing for decreasing the depression of the network, 2 - a system of two pumping stations pumping sewage to a common gravity subsystem, and $3-$ a system of two pumping stations located serially (operating conditions of a pumping station located closer to the water treatment plant may be dependent on the station located earlier).

\footnotetext{
* Corresponding author: i.nowogonski@iis.uz.zgora.pl
} 
Examples of the analysed gravity-pressure systems characterized by various levels of complexity pertain to theoretical examples [2], or ones based on design concepts [3] or existing systems.

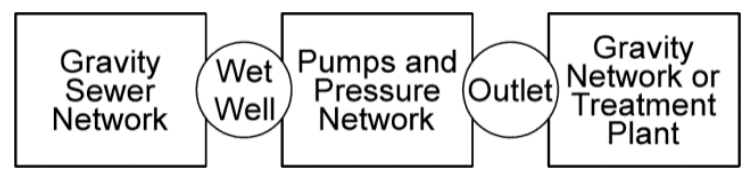

Fig. 1. Simplest pressure and gravity combined sewage systems [2].

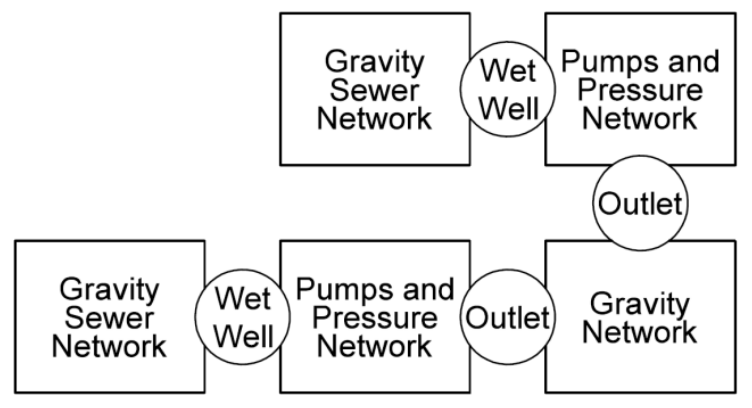

Fig. 2. Pressure and gravity combined sewage systems - parallel connection of the pumping station.

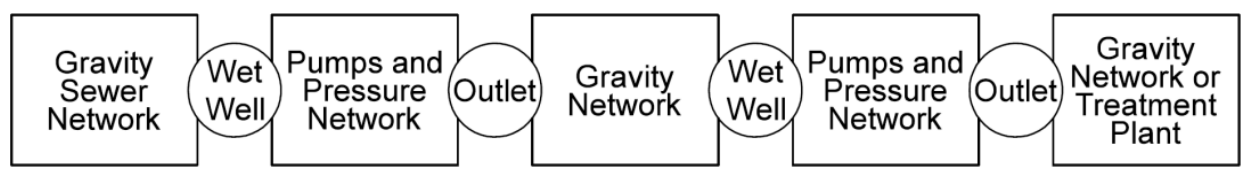

Fig. 3. Pressure and gravity combined sewage systems - serial connection of the pumping station.

The theoretical example [2] realized using Bentley Systems' WaterGEMS indicates the occurrence of backflows in the case of using the Epa SWMM computing engine. This most likely results from the specific nature of the software, requiring the application of the "Flap gate" feature at each segment of the pressure pipeline. Omission of protection against backflow results in the backup of sewage to the initial well (in Epa SWMM, each calculation node is treated as a well connected directly with the atmosphere). An example of the analysis of the concept of sewage discharge in the commune of Niegosławice [3] using the simulation model was verified by calculations realized using the Epanet programme. The obtained results made it possible to state that:

- average pump efficiencies are comparable in the majority of cases,

- in the case of pumping stations located at the end of a system (including the main pumping station discharging sewage to an existing system), there is an anomaly in the form of an unjustified increase in pump efficiency to the maximum value of pump efficiency input into the model,

- the mentioned anomaly may periodically lead to a distortion during the time of analysing the duration of an individual pumping cycle (rapid emptying of active volume).

As a result of the analysis of the collected information, it is usually possible to use the SWMM programme only in the case of stations pumping sewage via short pressure pipelines [4]. Channels with forced pressure flow are realized by:

- using cross-sections of the "Force main" type, making it possible to calculate hydraulic losses using the Darcy-Weisbach method, 
- introducing protection against backflow in each segment of the channel ("Flap gate" feature),

- introducing protection against the outflow of sewage onto the land surface ("Surcharge Depth" feature).

Controlling the start up / shut off of the pump may be done by:

- using the features of the pump ("Startup Depth" and "Shutoff Depth") or "Controls" procedures,

- using control instructions, enabling the start up / shut off of a pump based on the depth of sewage in the pumping station chamber.

More complicated systems, especially systems with a few stations pumping sewage to a common pipeline, are solved by applying tools usually used to analyse water distribution systems, such as Epanet, in which the pump in described using the following formula [5]:

$$
h_{i j}=-\omega^{2}\left(h_{0}-r\left(Q_{i j} / \omega\right)^{n}\right)
$$

where:

$h_{0}$ - the shutoff head for the pump;

$\omega$ - relative speed setting;

$r, n$ - the pump curve coefficients.

In consequence, the analysis of the frequency of the pumps starting up, probability of a few pumps starting up simultaneously and other issues which may be significant from the system operator's point of view is more difficult. The question of whether and to what extent such systems can be replicated in the model of the water sewage network without the loss of quality and reliability of the obtained results ought to be posed.

\section{Calculation examples}

\subsection{Example 1}

The calculation example accounts for a system with a single pump, pumping sewage to a pressure pipeline consisting of 5 calculation segments. The first segment ought to be treated as a pipeline within the pumping station, the remaining are treated as calculation segments, the characteristics of which stem from their location and natural topography. 


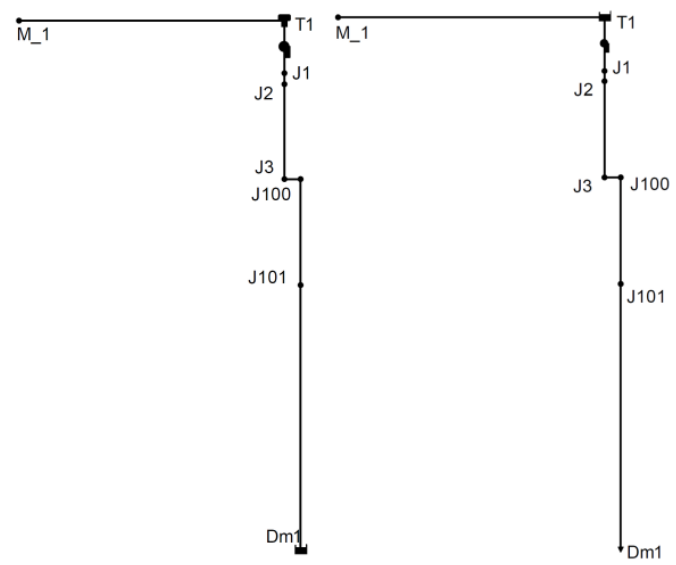

Fig. 4. Calculation schemes of pumping station in Example 1. (M_x - manhole, Tx - tank of pumping station, $\mathrm{Jx}$ - junction, Dmx - discharge manhole).

The model was recreated in two variants:

- protective measures against backflow in each segment of the channel as well as against sewage outflow onto the land surface were introduced - Variant I,

- in addition to the above changes, cross-sections of the "Force Main" type were applied Variant II.

The results were confronted with an identical system recreated using the Epanet programme. The gravitational inflow channel to the working chamber was simulated with a pipeline of larger diameter and "negative" demand. The schemes of connections prepared using the Epanet and Epa SWMM programmes have been presented in Figure 4.

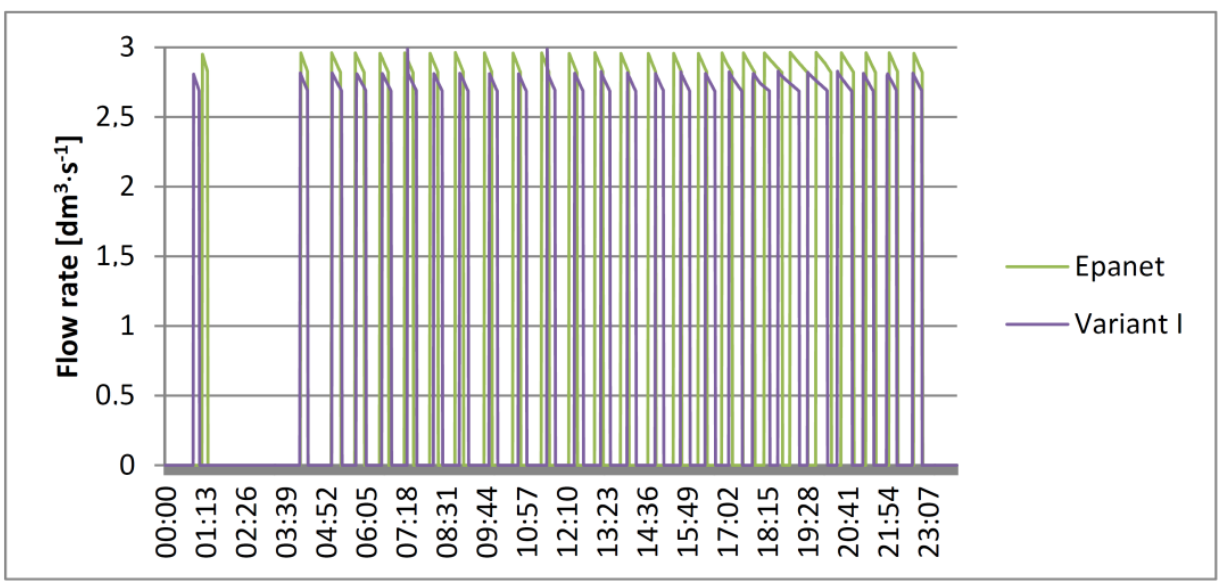

Fig. 5. Efficiency of analysed pump over the course of a given 24-hour period - Variant I. 


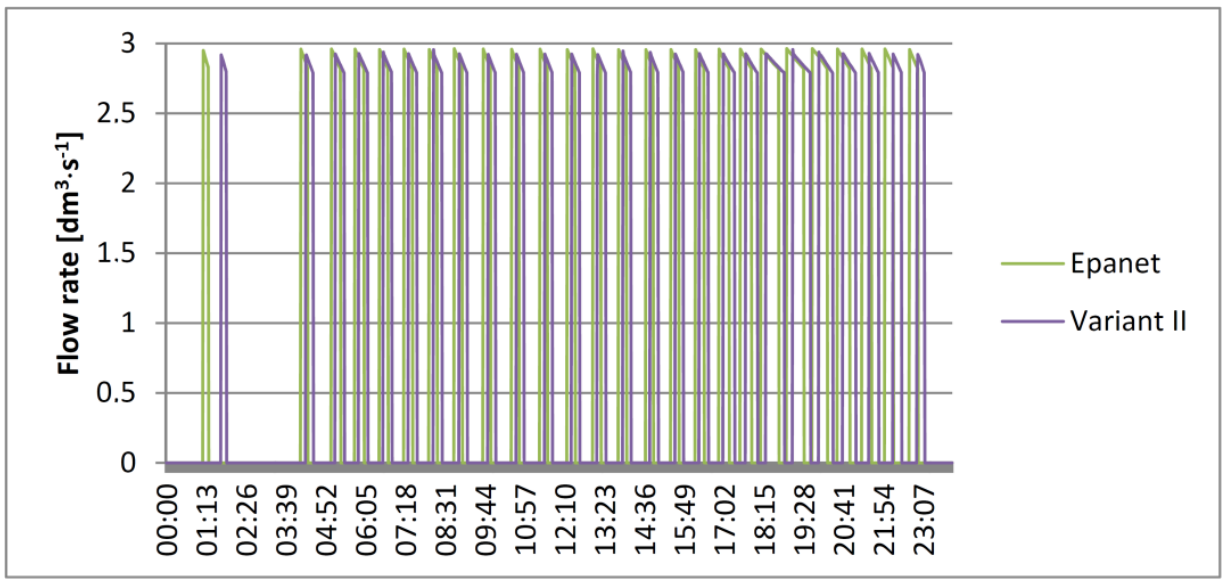

Fig. 6. Efficiency of analysed pump over the course of a given 24-hour period - Variant II.

In Figures 5 and 6, graphs showing the efficiency of a pump over time for both calculation variants have been shown in comparison to those obtained using the Epanet programme. Slight differences between pump efficiency in Figure 6 are connected with identical formulas of estimating flow resistance in both models. Omitting the "Force main" feature and carrying out all calculations based on the Manning Formula is connected with the occurrence of greater differences in the obtained results.

\subsection{Example 2}

The calculation example accounts for a network of two pumping stations (including one equipped with two working pumps) pumping sewage to a common pressure pipeline.

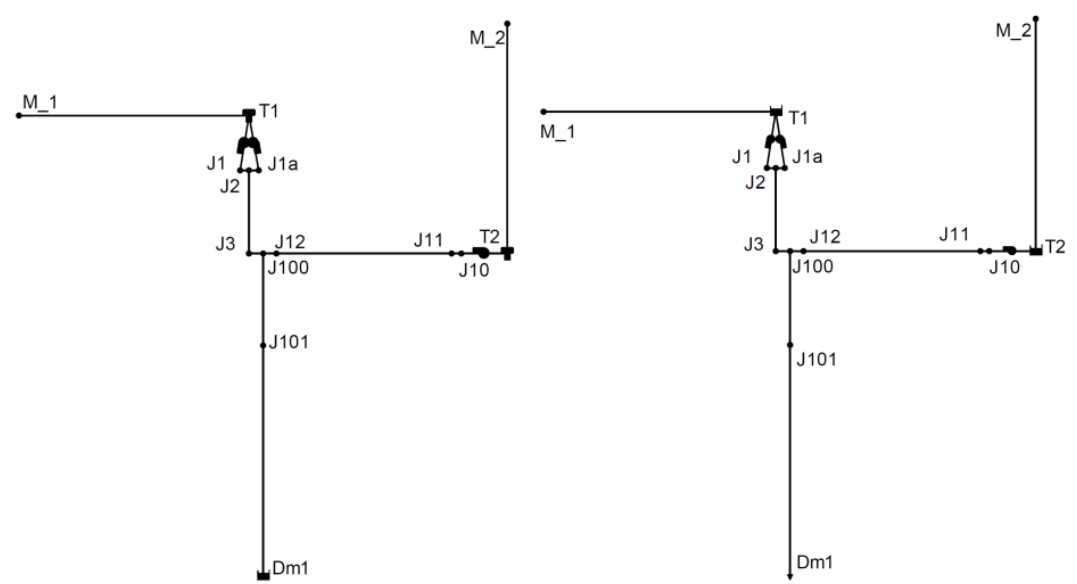

Fig. 7. Calculation schemes of networks in Example 2. (M_x - manhole, $T x-\operatorname{tank}$ of pumping station, $\mathrm{Jx}$ - junction, Dmx - discharge manhole). 


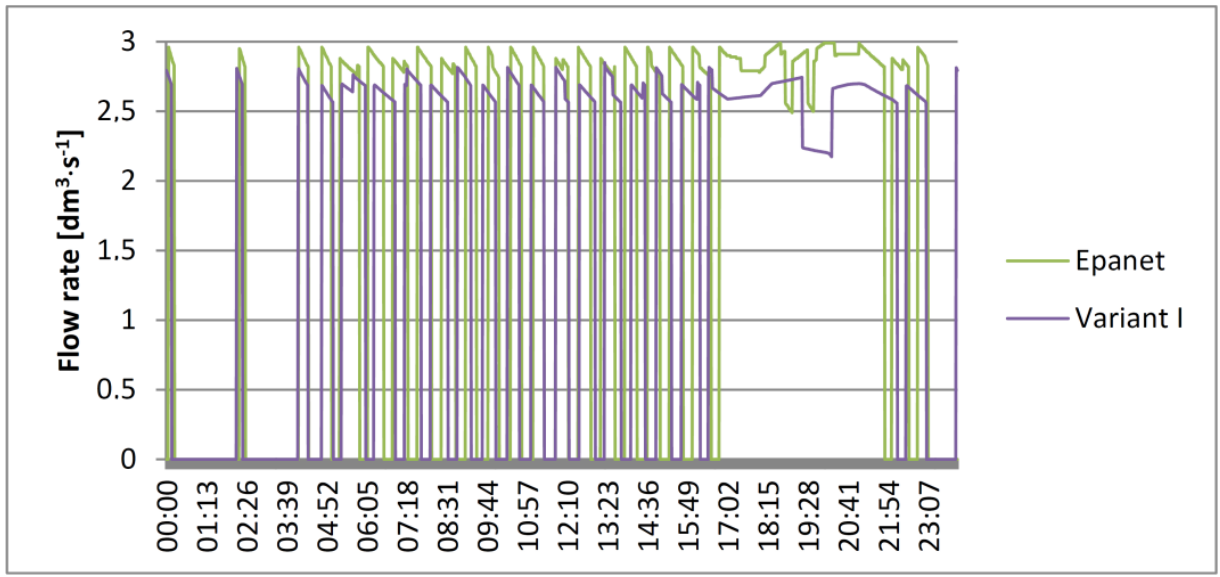

Fig. 8. Efficiency of main pump in pumping station with two pumps over the course of a given 24-hour period - Variant I.

In the analysed example, identical calculation variants as in Example 1 were carried out. The schemes of connections realized using the Epanet and Epa SWMM programmes have been presented in Figure 7.

Figures 8, 9 and 10 show graphs of the efficiency of individual groups of pumps over the course of a given 24-hour period for Variant 1, and Figures 11, 12, and 13 - for Variant II. All results were checked against results obtained using the Epanet programme. Also in this case, acceptable and comparable results were obtained under the condition that the Darcy-Weisbach form was used. Both the efficiency of pumps as well as the total running time of pumps over a given 24-hour period are comparable.

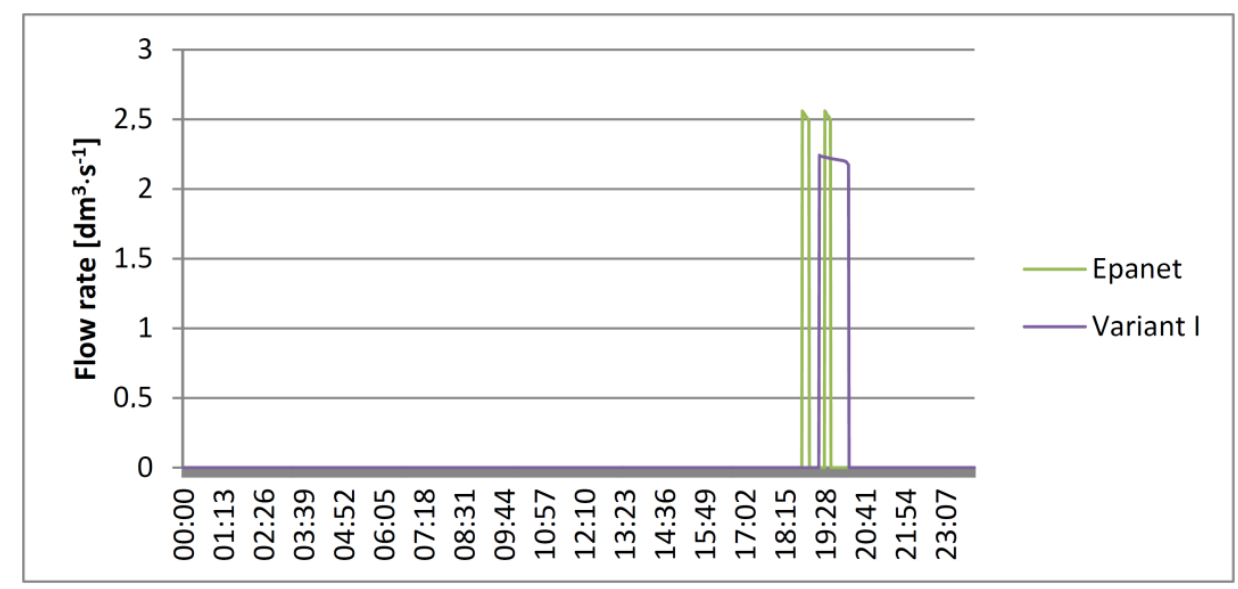

Fig. 9. Efficiency of auxiliary pump in pumping station with two pumps over the course of a given 24-hour period - Variant I. 


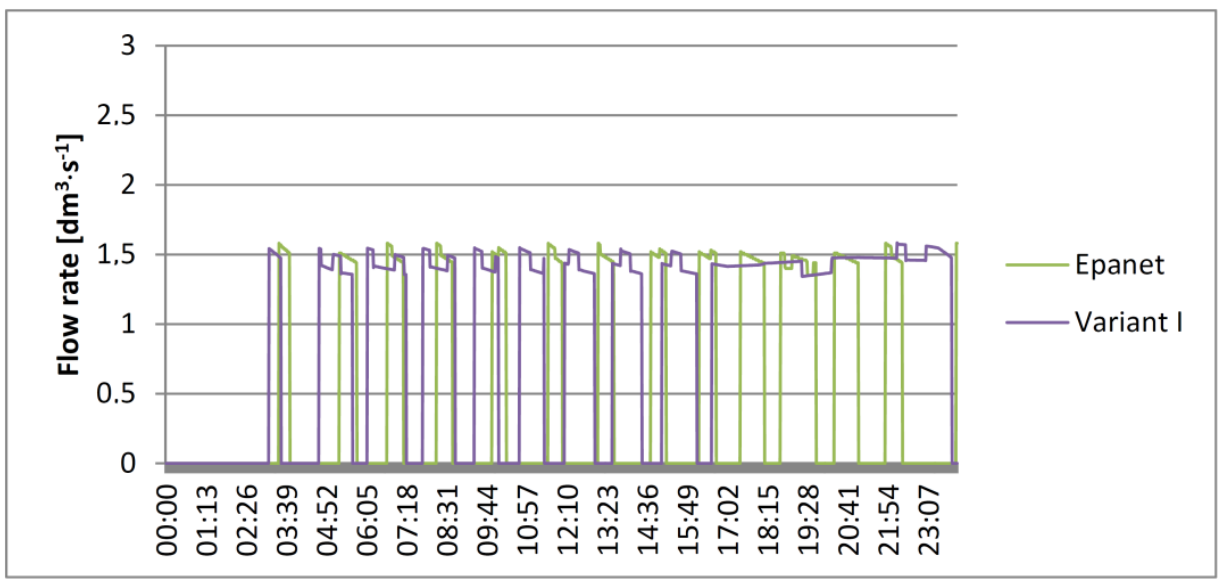

Fig. 10. Efficiency of pump in pumping station with one pumping engine over the course of a given 24-hour period - Variant I.

The only observable difference is in the case of all pumps running simultaneously in the event of the second pump being started up when the efficiency of a single pump is too low in relation to the inflow of sewage to the pumping station. Irrelevant of the accuracy of recreating the model, there is a one-time start-up of the pump in the case of the Epa SWMM, whereas using the Epanet model, the phenomenon is divided into two start-ups of significantly shorter duration.

\section{Discussion of results}

The following were selected as criteria for assessment: maximum pump efficiency, total running time of pump over a given 24-hour period and the number of times the pump started up over course of 24 hours. In the case of the simplest example in Calculation Variant II, the error of estimating efficiency does not exceed $0.2 \%$ and the error of estimating the running time of the pump is equal to $1.5 \%$. The number of start-ups is identical and amounts to 26, irrelevant of the applied model. Omitting the "Force main" feature leads to an increase in the value of: the error of estimating pump efficiency to $0.8 \%$, and the error for indicating the running time of the pump to $5.2 \%$. In this case, the Epa SWMM model anticipates decreasing the number of start-ups to 25 over the course of a 24-hour period.

In the case of Example 2, Variant II continues to provide acceptable values: error of estimating maximum efficiency at the level of $0.4 \%$ (T1-J1), $1.8 \%$ (T1-J1a) and $0.7 \%$ (T2-J10); error of estimating the running time of the pump of $0.1 \%$ (T1-J1), $35.1 \%$ and $3.3 \%$ (T2-J10). The number of start-ups using the Epanet model amounted to 23, 2 and 14, respectively; in the case of the auxiliary pump (T1-J1a) and single-pump pumping station (T2-J10), the values decreased by 1 start-up. In Variant 1: the error of estimating efficiency changed to respective values of $4.9 \%, 14.1 \%$ and $0.2 \%$, while estimation error of pump running time increased to values of $5.2 \%, 55.5 \%$ and $52 \%$. The number of start-ups decreased to 21,1 , and 10 . Values obtained for this variant are not acceptable. 


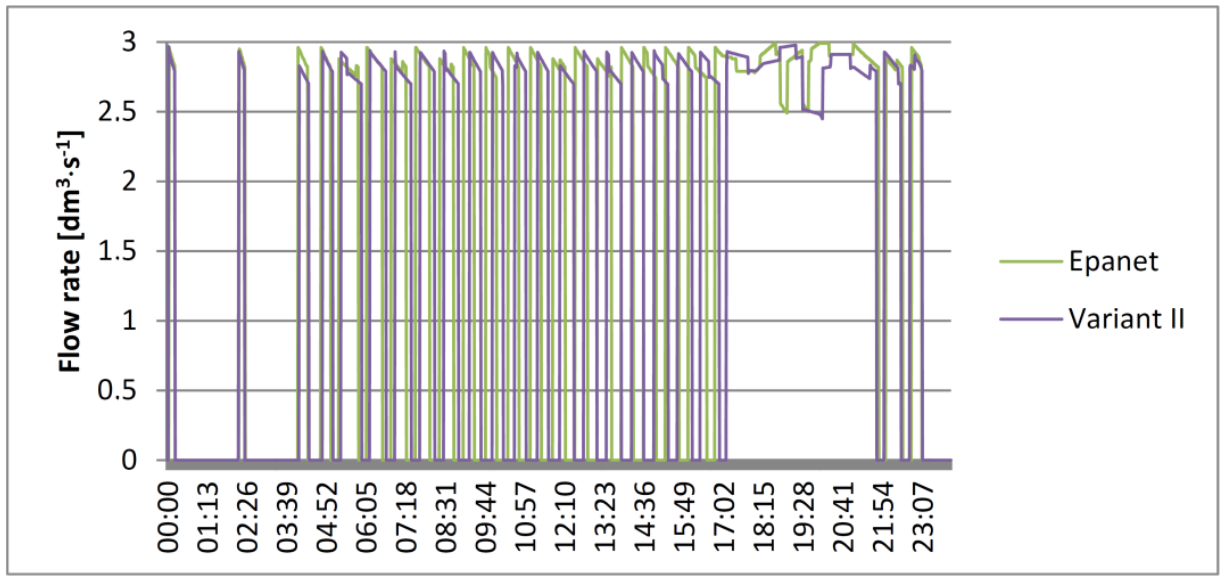

Fig. 11. Efficiency of main pump in pumping station with two pumps over the course of a given 24-hour period - Variant II.

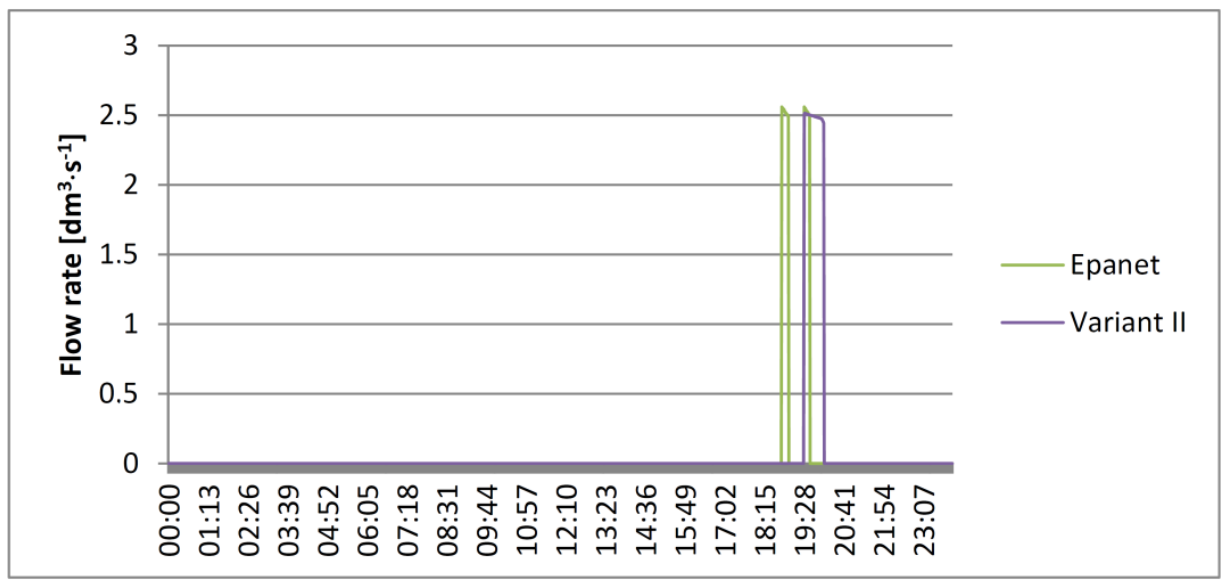

Fig. 12. Efficiency of auxiliary pump in pumping station with two pumps over the course of a given 24-hour period - Variant II.

As opposed to the example provided in literature [3], there was no necessity to replace the subsystem pump type 3 characteristics [4] - pressure pipeline, with type 4 characteristics [4]. The necessity to make such a simplification occurs mainly in the case of long pressure pipelines where more than one "high" point is present, where it is necessary to install an air relief valve. It is also not beneficial to locate an expansion well significantly below the last "high" point. In this case, both calculation-wise and operation-wise, one ought to consider the possibility of gravitational flow in the end segment of the distribution system. 


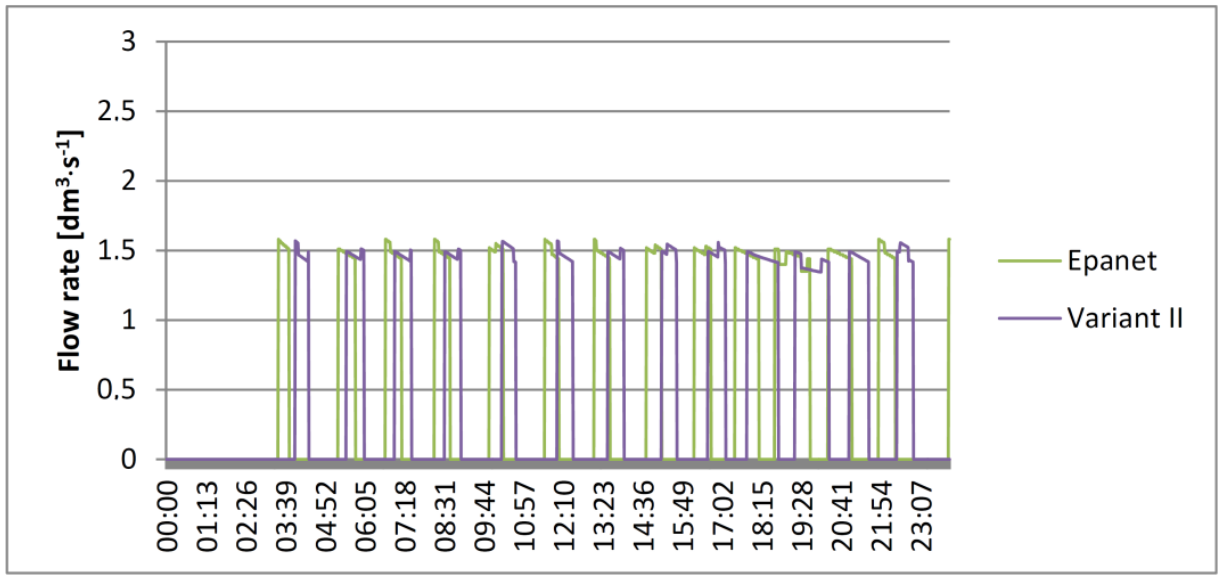

Fig. 13. Efficiency of pump in pumping station with one pumping engine over the course of a given 24-hour period - Variant II.

\section{Conclusion}

The analysis of the obtained simulation results and comparing the results obtained based on the Epanet and Epa SWMM programmes made it possible to draw the following conclusions:

- results obtained based on the Epanet and Epa SWMM programmes are comparable in the case of pressure pipelines located in areas of relatively even terrain,

- results are acceptable even in the case of a few stations pumping sewage to a common pressure pipeline assuming that all of the mentioned conditions are fulfilled,

- in the case of the occurrence of unstable results assuming a serial location of the pumping stations, one should consider replacing selected subsystems pump characteristics - pressure pipeline with type 4 characteristics.

\section{References}

1. J. Tian, J. Cheng, Y. Gong, Eng. Opt. 50, 1-11 (2017)

2. M. Jin, T. Walski, (11 $1^{\text {th }}$ ICUD, Edinburgh, Scotland, UK, 2008)

3. I. Nowogoński, E. Ogiołda, M. Kubiszyn, SR UZ EE (to be published)

4. L.A. Rossmann, US EPA, Washington DC, 40-62 (2017)

5. L.A. Rossmann, US EPA, Cincinati, OH 45268, 187 (2000) 